\title{
Metas académicas de los estudiantes de secundaria de la IED Pestalozzi jornada vespertina
}

\author{
Marlon Angulo Cetarez \\ Decanatura Académica, Escuela Naval de Suboficiales A.R.C. "Barranquilla”, Barranquilla, Colombia. \\ marlon.angulo@armada.mil.co
}

Recibido: Agosto 06, 2018.

Recibido en su versión corregida: Diciembre 10, 2018.

Aceptación: Abril 04, 2019.

Cómo citar: Angulo Cetarez, M. (2019). Metas académicas de los estudiantes de secundaria de la IED Pestalozzi jornada vespertina. Revista Sextante, 21, pp. 28 - 33, 2019.

\section{Resumen}

Esta investigación va orientada a comparar las metas académicas de hombres y mujeres de secundaria de la Institución Educativa Distrital Pestalozzi (jornada vespertina) de la ciudad de Barranquilla-Colombia, medidas a partir de los factores metas de aprendizaje, metas de logro y metas de refuerzo social. Son fundamentales los factores anteriores porque en el caso de la motivación tiene un efecto sobre la implicación del alumno en su proceso de aprendizaje (mucha implicación = afrontamiento activo; poca o nula implicación = afrontamiento pasivo), lo cual significa que cuanta más intensa y potente sea la intención de aprender, más se implicará en su aprendizaje, es decir, jugará un papel más activo y dedicará más tiempo y esfuerzo a estudiar y trabajar (Abellán, 2018, pág. 115).

Se recolecta información a través de un cuestionario realizado a un grupo de estudiantes seleccionados con muestreo aleatorio simple entre los 14 y 18 años, utilizando la metodología cuantitativa para procesarlos y analizar los resultados obtenidos. Este trabajo se desarrolla por etapas: inicia con la revisión de la literatura que concierne a los factores de aprendizaje, conociendo investigaciones previas llevadas a cabo en este campo y aterrizándolas en el marco teórico de esta investigación; un segundo momento expone la forma como se llevará a cabo la investigación a través del instrumento de medida (cuestionario) con el respectivo análisis estadístico; y por último, un análisis en la discusión, terminando la conclusión con las propuestas y futuros trabajos de investigación. En la referencia bibliográfica se indican textos, revistas y sitios web utilizados en este trabajo.

Palabras clave: Activos intangibles; Factores de aprendizaje, Motivación; TIC.

\section{Academic goals of the IED Pestalozzi high school students at evening session}

\begin{abstract}
This research is oriented at comparing the academic goals of men and women in high public-school Pestalozzi Educational Institution evening session of the city of Barranquilla-Colombia measured from the factors learning goals, achievement goals and goals of social reinforcement. The above factors are fundamental because in the case of motivation it has an effect on the student's involvement in their learning process (a lot of involvement $=$ active coping, little or no involvement $=$ passive coping), which means that the more intense and powerful the intention to learn the more will be involved in their learning, that is, he will play a more active role and dedicate more time and effort to study and work (Abellán, 2018).

Information is collected through a questionnaire made to a group of students selected with simple random sampling between 14 and 18 years using the quantitative methodology to process them and analyze the results obtained. This work is developed in stages: first, it begins with a review of the literature that concerns the learning factors, knowing previous research carried out in this field and landing them in the theoretical framework of this research; second, the way in which the research is carried out through the measuring instrument of a questionnaire with the respective statistical analysis and finally, an analysis in the discussion, ending the conclusion with the proposals and future research works. In the bibliographic reference texts, magazines and websites used in this work are indicated.
\end{abstract}

Keywords: ICT; Intangible assets; Learning factors; motivation. 


\section{Marco teórico}

El proceso de enseñanza llevado a cabo, tanto en el aula como fuera de ella, en diversas condiciones de aprendizaje, debe tener en cuenta los activos que hacen parte de lo intangible, uno de ellos es la motivación que tiene el estudiante para aprender los factores psicosociales, tales como: el afecto, el respeto entre otros que facilitan la aprehensión del conocimiento y ayudan al estudiante a alcanzar sus metas (Menchén Bellón, 2006).

Por otra parte, el ambiente físico influye significativamente en el aprendizaje del estudiante, ya que es un comunicador social en donde confluyen distintas representaciones, con la manifestación de las condiciones adecuadas para llevar a cabo este proceso junto con los símbolos que materializa, transmitiendo mensajes en lo espacial, es decir hace parte de la pedagogía invisible (González Quintián, 2006).

En realidad, existen una gran cantidad de factores que afectan significativamente el rendimiento de los estudiantes, los cuales deben ser tomados en cuenta para que se lleve a cabo un aprendizaje con sentido, en donde el joven se sienta a gusto con las actividades realizadas y le encuentre sentido a lo que está aprendiendo.

\section{Método}

El método empleado es de análisis cuantitativo con las respectivas ventajas que este conlleva (Hernández, Fernández, \& Baptista, 2010), con un estudio transversal de dos muestras independientes (hombres y mujeres) aplicando la prueba paramétrica $t$ de student para muestras independientes.

\section{Participantes}

En esta investigación participaron 30 alumnos de secundaria (15 hombres y 15 mujeres) de la Institución Educativa Distrital Pestalozzi, jornada vespertina, de la ciudad de Barranquilla-Colombia, con edades comprendidas entre los 14 y 18 años.

\section{Instrumentos}

Se utilizó la fuente de recolección de información primaria a través de un cuestionario estructurado de
20 preguntas, con una escala de 1 a 5 , donde 1 es nunca y 5 es siempre, la cual fue suministrada por UNINI en el doctorado en educación, en el módulo Factores de Aprendizaje, para medir tres factores (Clendenes, 2018): metas de aprendizaje (preguntas de la 1 a la 8), metas de logro (preguntas 9, de la 15 a la 20) y metas de refuerzo social (preguntas de la 10 a la 14), obteniendo el resultado de cada factor a través de la suma de los ítems correspondientes, los cuales a su vez permiten medir la variable dependiente: Escala de metas académicas (ver Anexo). Además, se empleó el software estadístico SPSS 22 para procesar y llevar a cabo el análisis de los datos recolectados.

\section{Procedimiento}

En la Institución Educativa Distrital Pestalozzi de la ciudad de Barranquilla-Colombia, en la jornada vespertina, a mediados del mes de julio de 2016, se empleó un M.A.S. (Muestreo Aleatorio Simple) en 3 salones de clases para seleccionar 3 grupos de 10 alumnos cada uno (5 mujeres y 5 hombres), se les explicó el objetivo de la encuesta y la importancia de conocer sus metas académicas. Se revisó la información recolectada para verificar que todas las preguntas fueron respondidas. En el tratamiento de la información se utilizó el software SPSS 22, tomando como parámetro de comparación la variable sexo.

\section{Análisis estadístico}

Inicialmente se realizó un resumen del procesamiento de los datos en cada una de las metas, para verificar que la información tabulada estuviese completa, es decir, detectar posibles casos de datos perdidos (ver Tabla 1).

En la anterior tabla se muestra que los datos recolectados están completos, es decir, no hay casos de valores perdidos.

En la Tabla 2 se observa que la media en las metas de aprendizaje y en las metas de logro fue similar, tanto para hombres como para mujeres, mientras que en las metas de refuerzo social se muestra una leve diferencia.

Seguidamente se verificó el supuesto de normalidad de cada factor en ambos grupos: hombre y mujer (ver Tabla 3) utilizando la prueba de 
Shapiro-Wilk al 5\% de nivel de significancia con las hipótesis:

$H_{o}$ : Los datos provienen de una distribución normal.

$H_{1}$ : Los datos NO provienen de una distribución normal.

La hipótesis nula $H_{o}$ se acepta cuando $\mathrm{P}$-valor $\geq$ $\alpha$, la hipótesis alternativa $H_{1}$ se acepta cuando Pvalor $<\alpha$.

El P-valor para las metas de aprendizaje fue de 0.194 en el caso de los hombres y de 0.601 en las mujeres; el P-valor para las metas de logro fue de 0.150 en los hombres y de 0.371 en las mujeres; el P-valor fue de 0.216 en los hombres y de 0.092 en las mujeres. Como el P-valor fue de $\geq 0.05$ tanto para hombres como para mujeres, en las variables metas de aprendizaje, metas de logro y metas de refuerzo social, se concluyó que los datos correspondientes a cada factor se comportaron con una distribución normal.

Posteriormente se corroboró el supuesto de igualdad de varianza de cada factor en ambos grupos: hombre y mujer (ver Tabla 4), utilizando la prueba de Levene al 5\% de nivel de significancia con las hipótesis:

$H_{o}$ : Los datos tienen varianzas iguales.

$H_{1}$ : Existe una diferencia significativa entre las varianzas de los datos.

El P-valor para las metas de aprendizaje fue de 0.522; el P-valor para las metas de logros fue de 0.456 y el P-valor para las metas de refuerzo social fue de 0.388. Como el P-valor fue $\geq 0.05$ para hombres y mujeres en las variables: metas de aprendizaje, metas de logro y metas de refuerzo social, se concluyó que cada factor presentó varianzas iguales.
Verificados los supuestos de normalidad y varianza se procedió a contrastar las medias de cada factor en ambos grupos: hombre y mujer (ver Tabla 5), utilizando la prueba $t$ de student al $5 \%$ de nivel de significancia con las hipótesis:

$H_{o}$ : NO existe una diferencia significativa en la media del factor entre el hombre y la mujer.

$H_{1}$ : Existe una diferencia significativa en la media del factor entre el hombre y la mujer.

Como P-valor fue $\geq 0.05$ para hombres y mujeres en las variables: metas de aprendizaje, metas de logro y metas de refuerzo social, se concluyó que cada factor presentó medias iguales y por lo tanto NO se observa una diferencia significativa.

\section{Resultados}

El análisis estadístico arrojó con la prueba $t$ de student similitud en las medias a un nivel de significativa del $5 \%$ entre los hombres y las mujeres, para los factores metas de aprendizaje, metas de logros y metas de refuerzo social, lo cual lleva a que NO existe una diferencia significativa al 5\% de nivel de significancia entre las metas académicas de los hombres y mujeres de la Institución Educativa Distrital Pestalozzi de la ciudad de BarranquillaColombia, en la jornada vespertina, para los estudiantes de noveno y décimo grado. Este resultado se valida con el cumplimiento de los supuestos de normalidad e igualdad de varianzas para los datos recolectados en el cuestionario.

\section{Discusión y conclusiones}

Los resultados obtenidos se pueden comparar con los puntos de vistas de autores como Pantoja, García-Valcárcel (García-Valcárcel, 1993), entre otros. Proponiendo posibles explicaciones a lo observado y, si existen diferencias, explicar las razones por las cuales se están presentando. 
Tabla 1. Resumen de procesamiento de casos

\begin{tabular}{cccccccc}
\hline & Género del & & \multicolumn{5}{c}{ Casos } \\
\cline { 3 - 8 } & estudiante & & Válido & & Perdidos & Total \\
\cline { 3 - 8 } & & $\mathbf{N}$ & Porcentaje & $\mathbf{N}$ & Porcentaje & N & Porcentaje \\
\hline Metas de & Hombre & 15 & $100 \%$ & 0 & $0 \%$ & 15 & $100 \%$ \\
aprendizaje & Mujer & 15 & $100 \%$ & 0 & $0 \%$ & 15 & $100 \%$ \\
\hline Metas de & Hombre & 15 & $100 \%$ & 0 & $0 \%$ & 15 & $100 \%$ \\
logro & Mujer & 15 & $100 \%$ & 0 & $0 \%$ & 15 & $100 \%$ \\
\hline Metas de & Hombre & 15 & $100 \%$ & 0 & $0 \%$ & 15 & $100 \%$ \\
aprendizaje & Mujer & 15 & $100 \%$ & 0 & $0 \%$ & 15 & $100 \%$ \\
\hline
\end{tabular}

Fuente: Los autores

Tabla 2. Media de los datos por metas.

\begin{tabular}{ccc}
\hline Metas & Género & Media \\
\hline \multirow{2}{*}{ De aprendizaje } & Hombre & 31.40 \\
\cline { 2 - 3 } & Mujer & 31.00 \\
\hline \multirow{2}{*}{ De logro } & Hombre & 30.20 \\
\cline { 2 - 3 } & Mujer & 29.87 \\
\hline \multirow{2}{*}{ De refuerzo social } & Hombre & 13.67 \\
\cline { 2 - 3 } & Mujer & 9.93 \\
\hline
\end{tabular}

Fuente: Los autores

Tabla 3. Prueba de normalidad.

\begin{tabular}{cccccccc}
\hline & Género del & \multicolumn{2}{c}{ Kolmogorov - Smirnov* } & \multicolumn{3}{c}{ Shapiro Wilk } \\
\cline { 3 - 8 } & estudiante & Estadístico & gl & Sig. & Estadístico & gl & Sig. \\
\hline \multirow{2}{*}{ Metas de aprendizaje } & Hombre & 0,148 & 15 & $0,2^{*}$ & 0,92 & 15 & 0,194 \\
& Mujer & 0,167 & 15 & $0,2^{*}$ & 0,955 & 15 & 0,601 \\
\hline \multirow{2}{*}{ Metas de logro } & Hombre & 0,192 & 15 & 0,143 & 0,913 & 15 & 0,15 \\
& Mujer & 0,162 & 15 & $0,2^{*}$ & 0,939 & 15 & 0,371 \\
\hline \multirow{2}{*}{$\begin{array}{c}\text { Metas de refuerzo } \\
\text { Social }\end{array}$} & Hombre & 0,171 & 15 & $0,2^{*}$ & 0,923 & 15 & 0,216 \\
& Mujer & 0,141 & 15 & $0,2^{*}$ & 0,899 & 15 & 0,092 \\
\hline
\end{tabular}

*. Esto es un límite inferior de la significación verdadera

a. Corrección de significación de Lilliefors

Fuente: Los autores

Tabla 4. Prueba de Levene para igualdad de varianza.

\begin{tabular}{cccc}
\hline & & \multicolumn{2}{c}{$\begin{array}{c}\text { Prueba t para la igualdad } \\
\text { de medidas }\end{array}$} \\
\cline { 3 - 4 } Metas de aprendizaje & $\begin{array}{c}\text { Se asumen varianzas iguales } \\
\text { No se asumen varianzas iguales }\end{array}$ & 0,42 & 0,522 \\
\hline Metas de logro & $\begin{array}{c}\text { Se asumen varianzas iguales } \\
\text { No se asumen varianzas iguales }\end{array}$ & 0,572 & 0,456 \\
\hline Metas de refuerzo social & $\begin{array}{c}\text { Se asumen varianzas iguales } \\
\text { No se asumen varianzas iguales }\end{array}$ & 0,768 & 0,388 \\
\hline
\end{tabular}

Fuente: Los autores 
Tabla 5. Prueba t de student para la igualdad de medias.

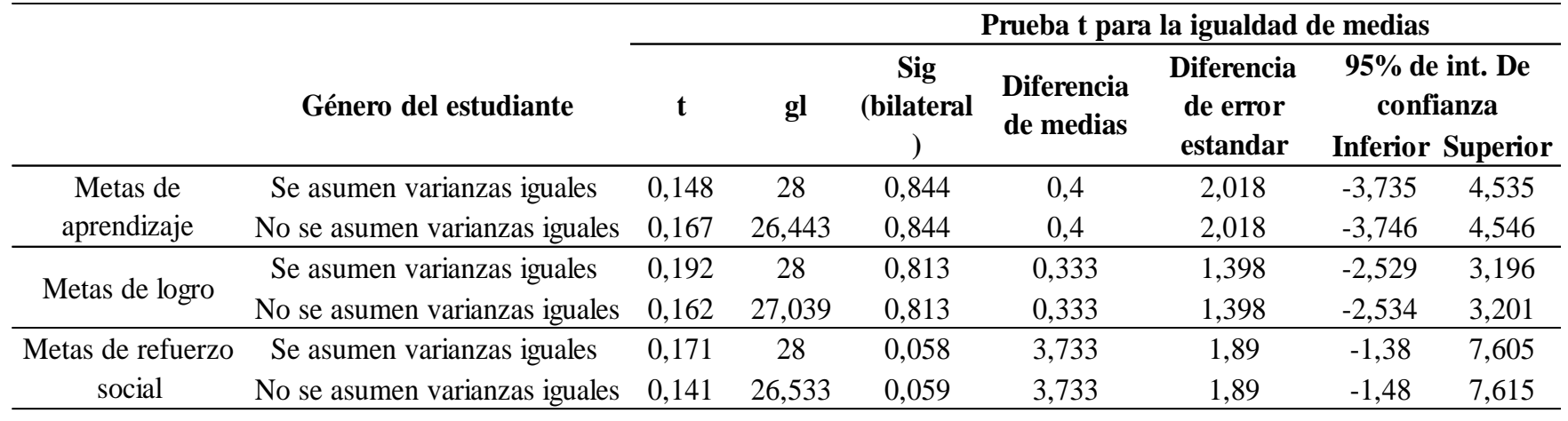

Fuente: Los autores

Este trabajo permite profundizar en los factores que influyen en las metas académicas como son: las metas de aprendizaje, de logro y de refuerzo social, identificando las características y potencialidades de los estudiantes, las cuales, a su vez, les sirven para una mejor selección de los estudios superiores a seguir y estar preparados para un mejor desempeño laboral: en el contexto laboral se requieren de trabajadores capaces de aprender, innovar y resolver problemas (García Valcárcel, 2008). Además, permite proponer posteriores investigaciones con el objeto de ampliar el campo de aplicación o de manejarla en otros ámbitos socioculturales según las necesidades que se presenten en la institución educativa.

Se recomienda realizar estudios referentes a otros problemas que se han detectado y que se presentan en la comunidad educativa, como son: el bajo nivel de compresión lectora, la falta de estímulo para el trabajo, el alto fracaso escolar, entre otros; para conocer qué tanto se relacionan con los factores estudiados en esta investigación.

\section{Referencias}

Abellán, L. (2018). Motivavión escolar y aprendizaje en la educación secundaria obligatoria. Ciencia \& futuro, 111-124.

Clendenes, C. (2018). Adecuada aplicación de los procesos pedagógicos para el logro de los aprendizajes. Lima.
García Valcárcel, A. (2008). La tutoría en la enseñanza universitaria y la contribución de las TIC para su mejora. Electrónica de investigación y evaluación educativa, 1-14.

García-Valcárcel, A. (1993). El proceso de enseñanza en las aulas universitarias. Qurriculum, 155-174.

González Quintián, C. (2006). La magia de los ambientes. Aljibe.

Hernández, R., Fernández, C., \& Baptista, P. (2010). Metodología de la investigación. México: Mc Graw Hill.

Menchén Bellón, F. (2006). El proceso creativo. Aljibe. 


\section{ANEXOS}

\section{Escala de metas académicas}

Edad:
Sexo
Hombre:
Mujer:

Calificación en lengua (0-10):

Calificación en matemáticas (0-10):

Responde en una escala de 1 a 5 , que oscila en un segmento de nunca a siempre las siguientes cuestiones, marcando con una X la casilla correspondiente.

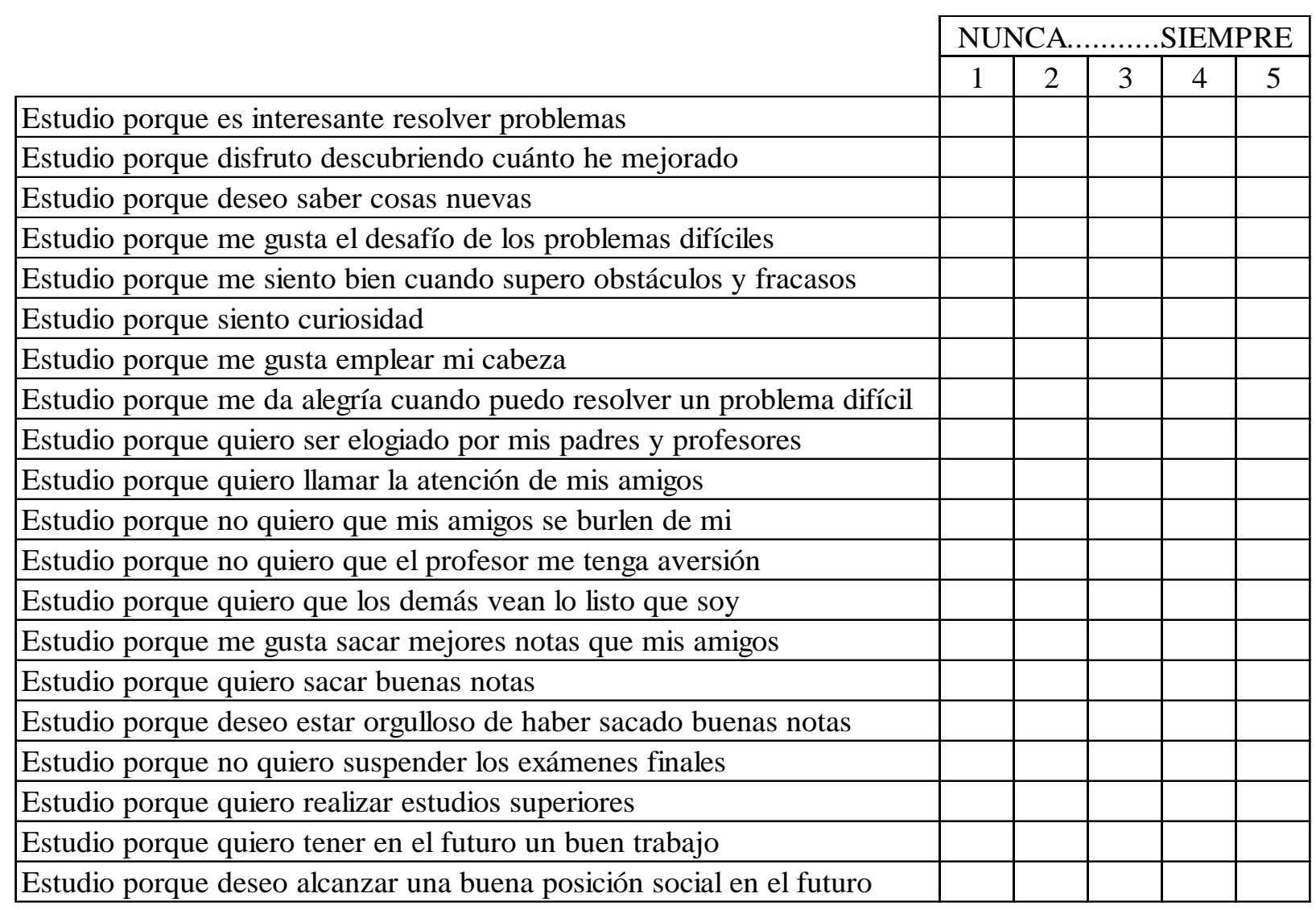

